\title{
Teorías del aprendizaje
}

\section{Theories of Learning}

\author{
Noemi Vega-Lugo ${ }^{a}$, Ruth Flores-Jiménez ${ }^{b}$ Ivette Flores-Jiménez ${ }^{c}$, Brenda Hurtado-Vega ${ }^{d}$ \\ José Sergio Rodríguez-Martínez ${ }^{e}$
}

\begin{abstract}
:
The theories of learning are a construction that explains and prophesies how the human being learns based on the conception of different theorists. Thus in a general way theories contribute to knowledge and from different approaches explain how the learning process occurs in human beings. They are those that perform the representation of a process that will allow a person to learn something. These will help us understand, anticipate and regulate behavior through the design of maneuvers that facilitate access to knowledge. In this way, it will interpret learning events and suggest solutions to problems that may arise in the teaching-learning process.

Within this conceptual map, five of the most representative education theories currently within psychology and education are addressed as they are; behavioral, constructivist, cognitive, socioconstructivist and social learning.
\end{abstract}

\section{Keywords:}

Learning theories, maneuvers of access to knowledge.

\section{Resumen:}

Las teorías del aprendizaje son una construcción que explica y profetiza el cómo aprende el ser humano basándose en la concepción de diversos teóricos. Así de una manera general las teorías contribuyen al conocimiento y desde diferentes enfoques explican el cómo se da el proceso de aprendizaje en los seres humanos. Son aquellas que realizan la representación de un proceso que permitirá a una persona aprender algo.

Estas nos ayudaran a entender, anticipar y regular la conducta a través del diseño de maniobras que facilitaran el acceso al conocimiento. De este modo interpretara los acontecimientos de aprendizaje y sugerirá soluciones a inconveniente que pueden surgir en el proceso de enseñanza- aprendizaje.

Dentro de este mapa conceptual se abordan cinco de las teorías de la educación más representativas actualmente dentro de la psicología y la educación como lo son; conductista, constructivista, cognoscitivista, socioconstructivista y aprendizaje social.

\section{Palabras Clave:}

Aprendizaje, teorías, maniobras de acceso al conocimiento.

\section{Introducción}

A lo largo de los años todo ha evolucionado y con ello la educación, las generaciones actuales traen consigo necesidades diferentes, y por ello nuevas formas de conocer, es por ello que la educación ha buscado herramientas que faciliten el proceso de enseñanza aprendizaje, y que va más allá de eso, formar alumnos capaces de hacerle frente a un mundo demandante y globalizado, es por ello la importancia que tiene para el docente conocer las teorías del aprendizaje y hacer uso ellas. .

\footnotetext{
a Autor de Correspondencia, Profesor Investigador de la Universidad Autónoma del Estado de Hidalgo, Instituto de Ciencias Económico

Administrativas. E-mail: noemivl@uaeh.edu.mx

${ }^{\text {b }}$ Profesor Investigador de la Universidad Autónoma del Estado de Hidalgo, Escuela Superior de Tlahuelilpan. E-mail: ruth@uaeh.edu.mx

c Profesor Investigador de la Universidad Autónoma del Estado de Hidalgo, Escuela Superior de Tlahuelilpan. E-mail: ivette@uaeh.edu.mx

d Maestra en Terapia Breve, Consultor Independiente. E-mail: brenda_sol1212@hotmail.com

${ }^{\text {e }}$ Profesor Investigador de la Universidad Autónoma del Estado de Hidalgo, Instituto de Ciencias Económico Administrativas. E-mail: 


\section{Tema principal}

Isabel García define el aprendizaje como todo aquel conocimiento que se adquiere a partir de las cosas que suceden en la vida diaria, de esto modo se adquieren conocimientos, habilidades, destrezas, aptitudes, etc. Esto se conseguirá a través de la experiencia, la observación y la instrucción.

Existen varias teorías en torno a por qué y cómo los seres humanos a continuación se describirán brevemente las citadas en el presente trabajo:

a) Conductismo: Es una corriente de la psicología que se centra en el comportamiento humano y animal, deja de lado todo lo intrínseco para concentrarse en las conductas observables y medibles. Esta conducta es determinada por refuerzos y castigos.

Se considera como fundador de esta teoría a J.B Watson, el nombro a la conducta su objeto de estudio, en ese momento la conducta que se estudiaba debía de ser cien por ciento observable y operacionalizable. Los fines que tenía el conductismo era la investigación y el análisis de las relaciones que rigen los sucesos ambientales y las conductas de los organismos en base a estimulo- respuesta.

- Constructivismo: Se puede entender como aquel que permite que el alumno construya su propio aprendizaje, las ideas principales de esta teoría son: el alumno es responsable de su propio conocimiento, construye su conocimiento por sí mismo, relaciona la información nueva con conocimientos previos, establece relaciones entre elementos, da significado a la información que recibe, necesita un apoyo pudiendo ser el profesor, pares o padres y el profesor se convierte en el orientador. Los principales estudiosos del constructivismo son: Piaget. y Ausubel.

- Cognoscitivismo: Los aportes de esta teoría se enmarcan en considerar al ser humano como ente pensante quien transforma el pensamiento como resultado de su ambiente interno y externo. De acuerdo como esta teoría la concepción de la enseñanza puede reducirse en los siguientes puntos: aprender y solucionar problemas, aprendizajes significativos con sentido, desarrollar habilidades intelectuales y estratégicas, proceso sociocultural, conocimiento previo y metas de aprendizaje.
- Aprendizaje social: Como principio de esta teoría se tiene que el aprendizaje directo no es el principal mecanismo de enseñanza, sino que el elemento social da la base de un aprendizaje nuevo en los individuos, esta teoría resulta útil para explicar cómo las personas aprenden y desarrollan nuevas formas de conducirse mediante la observación a otros individuos.

- Socioconstructivismo: Este paradigma fue desarrollado por L.S Vigotsky en la década de 1920, se basa en el alumno como ser social, donde la cultura modifica o crea todo su aprendizaje, si el conocimiento de una persona se construye socialmente de acuerdo a esta teoría será entonces necesario que la planeación se diseñe con actividades que permitan precisamente esa interacción social y que la relación no se centre en alumno-maestro, sino que se lleve a éste a su comunidad y se promueva la participación activa con sus pares.

\section{Conclusiones}

La educación a lo largo de la historia evoluciona dando respuesta a la necesidad de docentes y alumnos, por ello es de suma importancia el conocimiento de herramientas que permitan mejorar y optimizar el proceso de enseñanza- aprendizaje, el conocer las teorías de la educación permitirán mejora y conocer la manera en como los estudiantes aprenden y con esto coadyuvar a un aprendizaje significativo en el alumno.

\section{Referencias}

Alex, F. (2010). Psicología y Mente . Obtenido de https://psicologiaymente.com/psicologia/conductismo

Bisquerra, A. R. (2003). Modelos de orientación a intervención psicopedagógica. Barcelona : Praxis

Hernandez "Guanir", P. (2006). Educación del pensamiento y las emociones. Madrid.

Hernández, G. (1998). Paradigmas en Psicología de la educación. México : Paidós.

Machin Mastromatteo, J. (31 de 10 de 2017). Normas APA para la citación de documentos . Chihuahua, Chihuahua, México.

Woolfolk, A. (2006). Paradigma de la educación. México : Pearson. 


\section{Noemi Vega Lugo, Ruth Flores Jiménez, Ivette Flores Jiménez, Brenda Hurtado}

Vega, José Sergio Rodríguez Martínez.

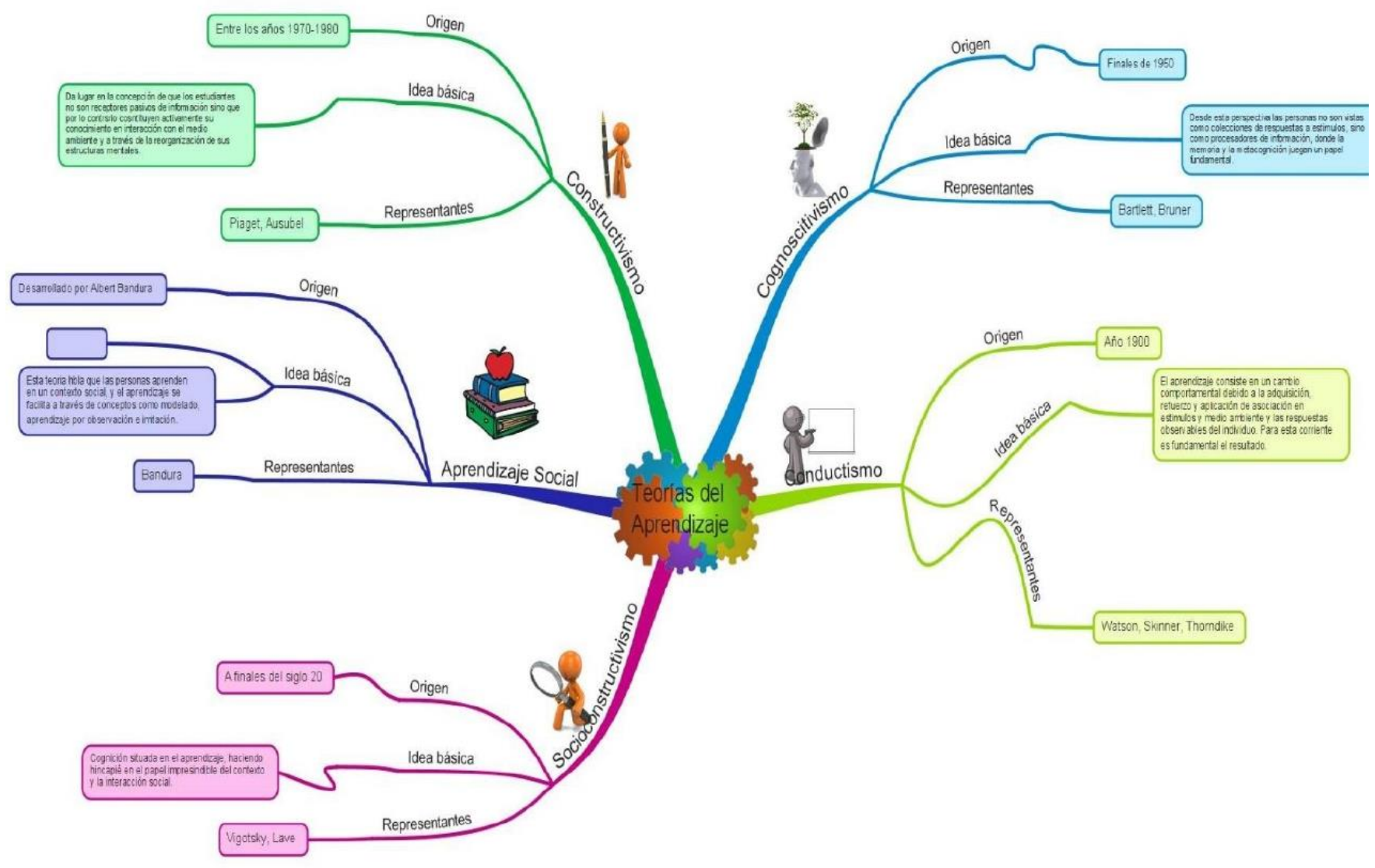

